\title{
Development of an inexpensive remote radio control system for slide projection
}

\author{
JAMES H. K. YOO \\ Department of Bioengineering, University of Texas, Health Science Center at San Antonio, San Antonio, \\ Texas 78284
}

\begin{abstract}
An inexpensive remote radio control system for slide projection has been developed. It consists of an electronic receiver/decoder designed for attachment to commercially available slide projectors and a self-contained, portable FM transmitter with a foldable (collapsible) antenna/pointer. The system allows remote lens focusing (forward and backward) and slide change (forward and reverse) to be accomplished over a 100 -ft range. It offers desirable freedom of movement to the lecturer or behavioral researcher, by eliminating locational restraints imposed by the extension cord.
\end{abstract}

Slide projectors are used by thousands of orators and researchers throughout the world It is often desirable to control slide projectors remotely without the restraint of extension cords. This is not only true for those public speakers and presentors on the podium, but also for many of the behavioral researchers (Humphries, Phoebus, \& Globus, 1974; Mackay, 1970). The system described here will free the speaker or operator from locational restraints and allow him to move freely or to perform necessary covert operations remotely.

An electronic receiver to be attached to commercially available slide projectors and a portable FM transmitter with a foldable pointer/antenna have been designed and constructed. The remote radio control system for the slide projector can transmit the control signals more than $100 \mathrm{ft}$. It has four functions: lens focusing, forward and backward, and slide change, forward and reverse. This paper describes the electronic circuit aspects of the inexpensive remote radio control system.

The overall functional block diagram of the system is given in Figure 1. Figures 2 and 3 describe the actual electronic circuitries of the tone generator and the remote control transmitter, and the receiver and signalprocessing units, respectively. The signal-sending unit is composed of function-selection switches, tone generator, and frequency-modulated transmitter. One of the four functions is selected by depressing the fingertouch switches. These activate the tone generator, which utilizes RCA MOS CD4046AE. Four different tones, ranging from $150 \mathrm{~Hz}$ to $1,300 \mathrm{~Hz}$, are generated by choosing different feedback resistances and are then fed to the FM modulated transmitter through a $.01-$ microF coupling capacitor. The tone signal is then fed to the varicap diode GE90 to modulate the reversebiased junction capacitance. This produces frequency deviation of the oscillation, due to changed resonant frequency of the tank circuit of the Hartley oscillator, which is embodied with a field-effect transistor ECG132. The center frequency of the transmitter is adjusted by a trimmer capacitor of $3.5-12 \mathrm{pF}$ to locate it at the quietest frequency in the $\mathrm{FM}$ band, from $88 \mathrm{MHz}$ to $108 \mathrm{MHz}$. The tank resonance coil is composed of 51/2 turns of No. 12 copper wire. Two choking coils of $1.0 \mathrm{microH}$ and $5 \mathrm{microH}$ are used to confine the high-frequency currents to the ECG 132 oscillation circuit and to allow the dc and tone-frequency currents to pass freely.

To extend the battery life, the $9 . \mathrm{V}$ battery is applied only when one of the tone-selector switches is activated. The power supply voltage for the FET oscillator ECG 132 is reduced to about $3 \mathrm{~V}$ by a Zenor diode 1N957. This may be simplified by using a 4.5 - or $5-\mathrm{V}$ battery instead. A $.5-\mathrm{m}$ foldable pointer is modified to function as an antenna. It is connected to the lower $1 / 2$ turn of the tank resonance coil. The assembled package of the transmitting unit is about 1.5 in. $x 2$ in. $x 4$ in. in size and is easily held in the hand. It is light enough to use as a pointer.

The receiving unit is composed of a bare-bone FM receiver, frequency-tuning adjuster, tone decoder and filter, and relay activator for the slide projector. A commercially available bare-bone FM receiver was used
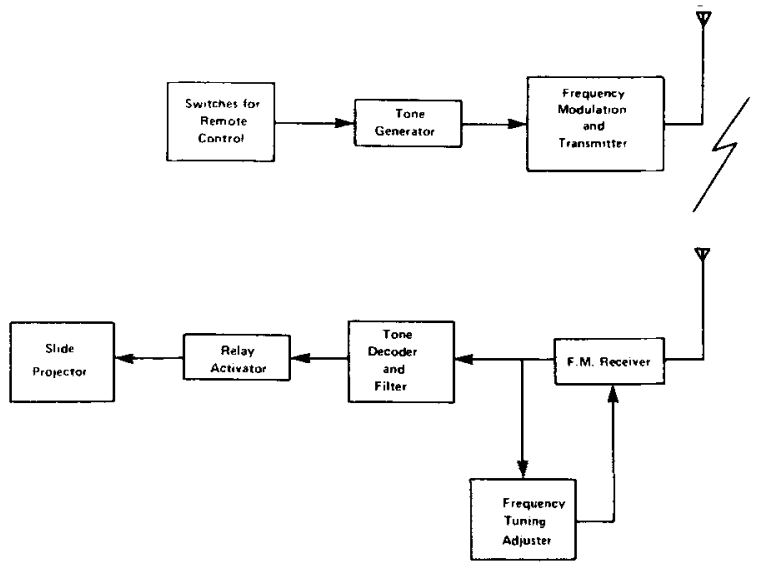

Figure 1. Functional block diagram of the remote radio control system for slide projection. 


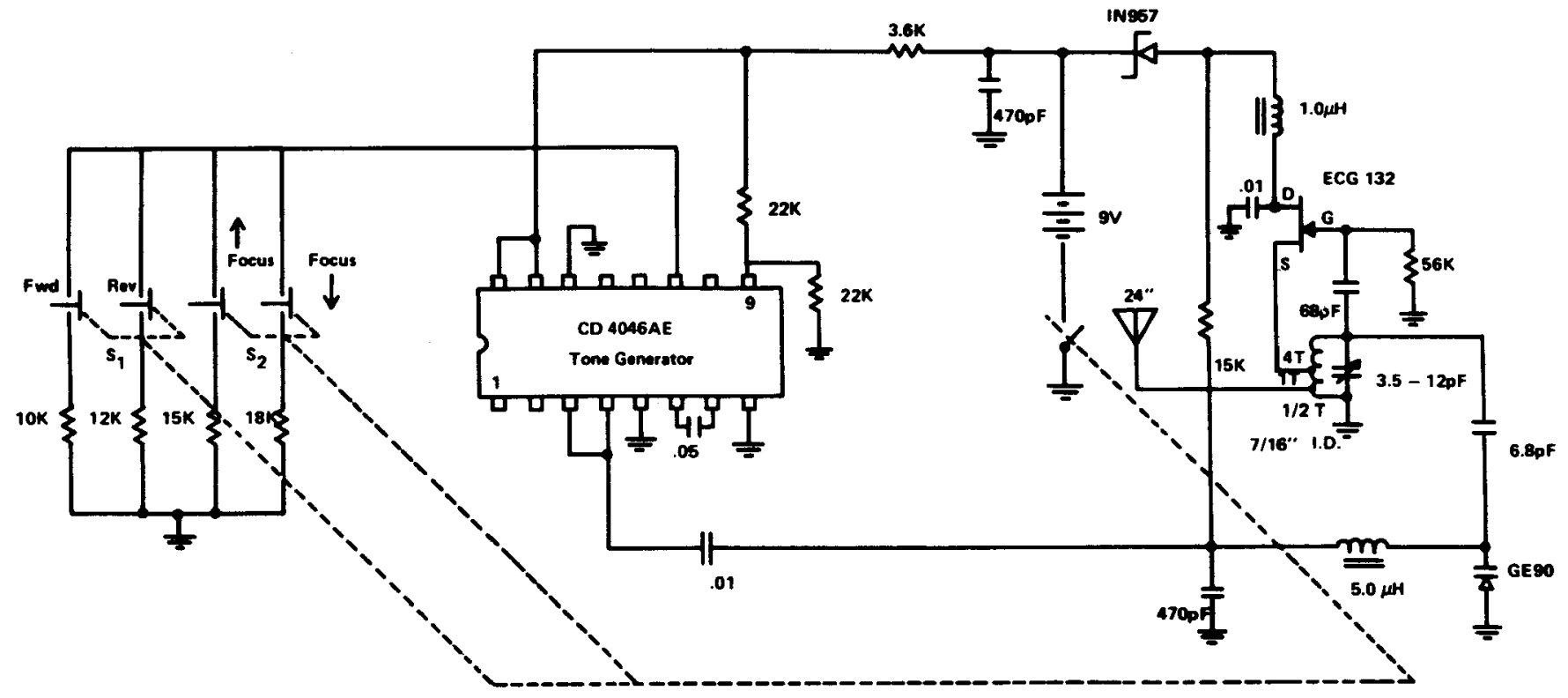

Figure 2. Schematic circuitry of the remote control transmitting unit.

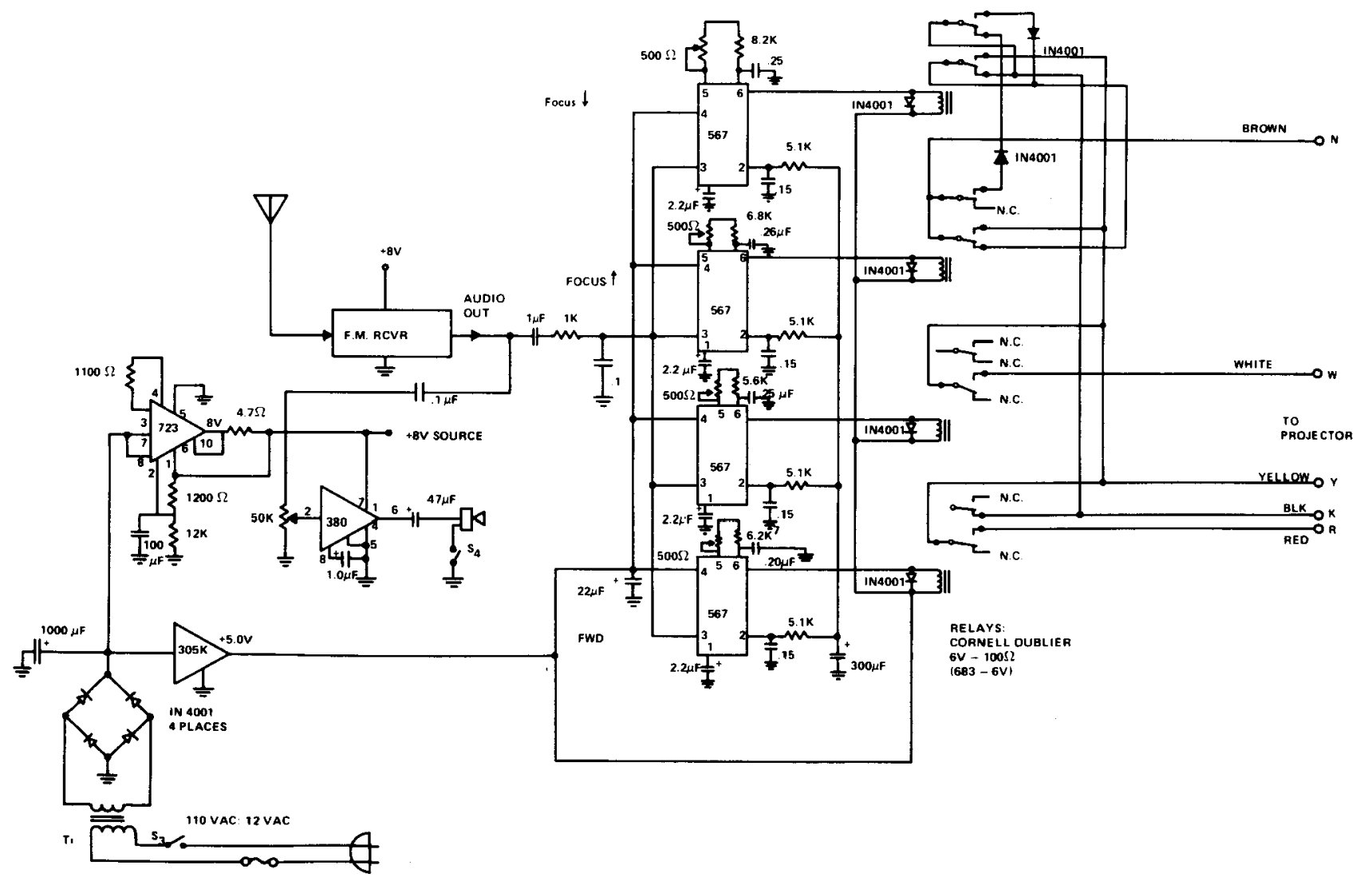

Figure 3. Schematic circuitry of the receiver and tone-decoder unit. 
for the study and cost about $\$ 7.50$. A 1-ft rod antenna picks up the FM control signal. The audio output from the receiver is fed to the tone-decoder unit, composed of four parallel phase-locked loop tone decoders (Gardner, 1966). NE567 tone-decoder phaselocked loop circuits separate the received control signals and activate the appropriate relay. 1N4001 diodes are connected in parallel with the relay coils to activate them selectively only one at a time. The relays are double-pole double-throw types made by the Cornell Dublier Company. They are activated selectively one at a time by 5.V dc supplied by Pin 6 of NE567. The proper circuit connections to meet Kodak 2 by 2 slide projectors are shown in Figure 3. The modified slideprojector extension cord and male sockets are plugged into the slide projectors as usual.

An additional feature of the system is the frequencytuning adjuster, composed of LM380 and a minispeaker. The audio output from the FM receiver is fed to LM380 to accomplish further amplification for audible sound. The receiver tuning is achieved with maximum speaker sound and proper activation of the slide-projector activation switches in the chosen FM band. The unregulated power supply is provided by a four-diode bridge rectifier. A $+5-\mathrm{V} d c$ is obtained at the output of the voltage regulator $\mathrm{LM} 305 \mathrm{~K}$. The regulated $+8 \cdot \mathrm{V} \mathrm{dc}$ is provided by another voltage regulator, LM723, and the associated low-pass filter. This may be easily replaced by different types of regulators, such as LM320-8T. The components for construction of the receiving unit are commercially available off-shelf components. They may cost less than $\$ 25$. The size of the assembled prototype is about 6 in. $x 8$ in. $x 8$ in. It is portable and has a leather carrying handle on top of the unit.

Installation and field operation of the remote radio control system for slide projection are simple. The system is nondirectional in its $100-\mathrm{ft}$ coverage. Operational range may be extended farther. Since the output is smaller than $50 \mathrm{~mW}$, it may not need to meet any stringent FCC license requirements. The flexibility of choosing the proper FM broadcasting frequency for the quietest band in the region is a very important aspect of the system. Interference seldom occurred during the extensive testing operations. Accurate tone generation by a MOS IC and reliable decoding by phaselocked loop tone-decoder circuitry, along with the low cost, may offer very useful applications in many disciplines of behavioral research.

\section{REFERENCES}

Gardner, F. M., Phase-lock techniques. New York: John Wiley, 1966.

Humphries, J., Phoebus, E., \& Globus, G. A micropower multichannel biotelemetry system. Psychophysiology, 1974, 11, 382-387.

MACXAY, R. S. Biomedical telemetry. New York: John Wiley, 1970.

(Received for publication September 24, 1976; accepted October 20, 1976.) 